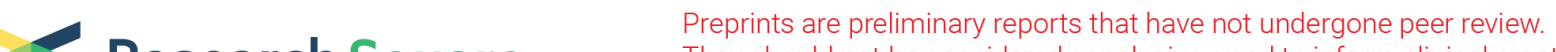 s.
}

\section{A Long Way Home. Biventricular Impella®, VA- ECMO and Extracorporeal LVAD as Bridge to a Permanent LVAD in Severe Ischemic Cardiogenic Shock: A Case Report}

\section{Monika Sadlonova}

University Medical Center Göttingen: Universitatsmedizin Gottingen

\section{Birgit Gerecke}

University Medical Center Göttingen: Universitatsmedizin Gottingen

\section{Aschraf El-Essawi}

University Medical Center Göttingen: Universitatsmedizin Gottingen

\section{Lars-Olav Harnisch}

University Medical Center Göttingen: Universitatsmedizin Gottingen

\section{Onnen Moerer}

University Medical Center Göttingen: Universitatsmedizin Gottingen

\section{Konrad Meissner}

University Medical Center Göttingen: Universitatsmedizin Gottingen

\section{Claudius Jacobshagen}

University Medical Center Göttingen: Universitatsmedizin Gottingen

\section{Hassina Baraki}

University Medical Center Göttingen: Universitatsmedizin Gottingen

Ingo Kutschka ( $\square$ ingo.kutschka@med.uni-goettingen.de)

University Medical Center Göttingen: Universitatsmedizin Gottingen

\section{Case report}

Keywords: cardiogenic shock, VA-ECMO, biventricular impella, LVAD, case report, multidisciplinary expertise

Posted Date: September 23rd, 2020

DOl: https://doi.org/10.21203/rs.3.rs-78251/v1

License: (9) This work is licensed under a Creative Commons Attribution 4.0 International License. Read Full License 


\section{Abstract}

Background: A severe cardiogenic shock can present with clinical complications such as arrhythmias, ischemia and organ failure and even today is associated with a high mortality. Extracorporeal life support (ECLS) by veno-arterial extracorporeal membrane oxygenation (VA-ECMO), Impella ${ }^{\circledR}$ and other mechanical circulatory support systems can reduce the acute circulatory failure.

Case presentation: We present the case of a 38-year-old woman with an acute heart failure due to a coronary artery disease who underwent emergency coronary artery bypass grafting and intraoperative implantation of a VA-ECMO. Over the next 4 months, a multidisciplinary team-approach bridged the patient using first a left ventricular (LV) support system (Impella), then additionally a right ventricular (RV) Impella and finally a temporary paracorporeal continuous flow left ventricular support (Rotaflow). Following a promising neurological recovery, a long-term left ventricular assist device (LVAD) was implanted in a bride to transplant (BTT) concept.

Conclusions: The addition of LV Impella and RV support by Impella (BiPELLA) on top of VA-ECMO may support survival of patients with refractory cardiogenic shock. In complex biventricular heart failure, an expert center must be able to provide an early multi-modular intervention with elaborated mechanical circulatory support due to a multidisciplinary expertise.

\section{Learning Points}

- In severe cardiogenic shock due to biventricular cardiac dysfunction, a combined extracorporeal life support (ECLS) with left and right ventricular Impella on top of veno-atrial extracorporeal membrane oxygenation (VA-ECMO) can successfully be installed as a bridge to implantation of a permanent left ventricular assist device (LVAD).

- A staged approach to ventricular support may facilitate decision-making especially in the setting of an unclear neurological status and insufficient left ventricular unloading by VA-ECMO.

- In complex biventricular heart failure, an expert center must be able to provide an early multi-modular intervention with differentiated mechanical circulatory support and individually adapted (high risk) procedures due to a multidisciplinary expertise.

\section{Background}

Cardiogenic shock (CS) is the clinical presentation of circulatory failure with multiorgan dysfunction complicated by hemodynamic, cellular and metabolic abnormalities [1,2]. The most frequent cause of CS is acute myocardial infarction (AMI) accounting for $80 \%$ of cases in registries [2]. In the treatment of severe CS temporary mechanical circulatory support such as veno-atrial extracorporeal membrane oxygenation (VA-ECMO) or Impella ${ }^{\circledR}$ can be used as a bridge to recovery (BTR), as a bridge to an implantation of a left ventricular assist device (bridge-to-bridge, BTB), as bridge to decision (BTD) or 
sometimes as a bridge to transplantation (BTT) [1,3-5]. Few studies have shown that the addition of an Impella ${ }^{\circledR}$ device to a VA-ECMO ('ECPELLA' or 'ECMELLA') was associated with improved survival of patients with refractory $C S[6,7]$. There are small series with different proposals of unloading not only the LV Impella as an ECPELLA or ECMELLA concept, but also with a left ventricular (LV) and a right ventricular (RV) Impella (BiPELLA) or a prolonged Impella (PROPELLA) with improved survival outcomes [8]. In view of the increasing therapeutic options, a multidisciplinary expert-team approach to patients in cardiogenic shock becomes highly important $[9,10]$.

\section{Case Presentation}

A 38-year-old woman was referred to our institution from a peripheral hospital with progressive dyspnea for months with aggravation in the last three weeks, and bilateral pleural effusions (Table 1; Figure 1); no symptoms of angina, negative family history, risk factor of moderate smoking for many years.

Echocardiography revealed a severely reduced LV function with an ejection fraction of $10 \%$ with global hypokinesia and apical akinesia and a moderately reduced right ventricular function. Moderate mitral and tricuspid regurgitation were documented. Initial hemodynamic support with dobutamine and noradrenaline was stopped driven by a beginning ischemia of the feet. Under the suspicion of an incipient pneumonia, blood cultures were collected and an antibiotic therapy initiated. Cardiac catheterization revealed a severe three-vessel coronary artery disease. Emergent coronary artery bypass grafting was performed with a left internal mammary artery graft to the left anterior descending artery, a sequential venous graft to a marginal branch and the marginal branch of the right coronary artery as well as a sequential venous graft to the right coronary artery and the posterior descending artery. As weaning from cardiopulmonary bypass was unsuccessful a peripheral (femoral) VA-ECMO was implemented. 
Table 1

Timeline of the case report

Presentation

Initial

treatment

The 38-year-old patient was transferred from a peripheral hospital with progressive dyspnea, pleural effusions on low dose inotropes. Echocardiography showed a severely reduced left ventricular function (LV) with an ejection fraction (EF) of $10 \%$.

Cardiac catheterization showed a severe three-vessel coronary artery disease. The patient received an emergency coronary artery bypass graft surgery with heart-lung machine (HLM) and VA-ECMO was established.

Extensive fluid volume substitution and vasoactive therapy.

Day 1

Day 4

Episodes of pulseless, polymorphic and monomorphic ventricular tachycardia. Continuous renal replacement therapy initiated due to acute kidney injury. Diagnosis of dyslipidemia with high levels of $L p(a)$.

Day 6-8

Day 8

Repeated episodes of ventricular fibrillation following the attempt to reduce ECMO support. More than 70 defibrillations and multiple antiarrhythmic drug therapies (magnesium, amiodarone, lidocain and ajmaline).

Our multidisciplinary heart-team decided to implant a LV Impella $\left(\mathrm{CP}^{\circledR}\right.$, Abiomed $)$ for LV unloading.

Day 9

Implantation of a right ventricular Impella $\left(\mathrm{RP}^{\circledR}, \mathrm{Abiomed}\right)$ to improve $\mathrm{RV}$ failure and prepare LVAD implantation. (Figure 2)

Day 10

Due to extensive hemolysis and an unclear neurological status under biventricular Impella support, we decided to implant a temporary extracorporeal left ventricular assist device (Rotaflow ${ }^{\circledR}$, Marquet) as a second bridge to long-term. At the same time, we removed the VA-ECMO and LV Impella $\left(\mathrm{CP}^{\circledR}\right.$, Abiomed) (Figure 3 ).

\section{Day 13}

Day 19

Removal of RV Impella ( $\mathrm{RP}^{\circledR}$, Abiomed).

After the patient had shown adequate neurological reaction implantation of longterm LVAD (HeartMate $3^{\mathrm{TM}}$, Abbott, USA) as a BTT-concept (Figure 4).

Thrombectomy of the right femoral artery with patch plastic after removal of the arterial cannula of the EMCO.

Day 23

Computer tomography of the brain showed no focal abnormalities preparing first uneventful extubation.

Day 35

Ventricular fibrillations for nearly 20 minutes with re-intubation and mechanical ventilation, 12 defibrillations, multiple antiarrhythmic therapies and cardiopulmonary resuscitation for 20 minutes. Coronary angiography showed open venous coronary artery bypass grafts but occluded LIMA graft (scared target area).

The hemodynamic situation improved. Catecholamine therapy could be terminated.

Epistaxis with nasal tamponade, second successful weaning and extubation.

Day 37

Day 36-40
Acute drop in blood pressure, bradycardia with cardiopulmonary resuscitation for approximately 30 minutes. Ventricular fibrillation with the need of intubation and defibrillation. On bronchoscopy multiple clots in trachea that led to hypoxia. 

on weaning, cerebral CT showed no focal abnormalities in the brain parenchyma.

Day 43-44

Day 72

Day 95

Day 114 Implantable cardioverter defibrillator (ICD) was implanted.

Amputations of I/IV digits of the left feet and debridement of catecholamine induced necrotic tissue from both feet.

The patient was transferred to a rehabilitation hospital with only minor neurological impairment with complete mobility of the right arm. Renal function had recovered.

The patient was discharged home.

\section{Day 154}

Extensive laboratory screening for vasculitis and rheumatologic causes revealed no abnormalities. However, a dyslipidemia with significantly increase lipoprotein (Lp) (a) was detected. Postoperatively the patient developed acute kidney injury with the need for continuous renal replacement therapy (CRRT).

During the next days, the patient suffered repeated episodes of malignant ventricular arrhythmias requiring more than 100 defibrillations and multiple antiarrhythmic therapies including magnesium, amiodarone, lidocaine, ajmalin and propranolol. LV-function remained poor and reduction of ECMO flow failed.

Following a new prolonged episode of refractory ventricular arrhythmias on the $7^{\text {th }}$ postoperative day the decision to unload the left ventricle by implantation of a left ventricular Impella (Impella CP ${ }^{\circledR}, A b i o m e d$ ) was made.

Uncertainty regarding sufficient right heart function subsequently led to implantation of a right ventricular Impella (Impella RP ${ }^{\circledR}$, Abiomed) to prepare the implantation of an of a permanent left ventricular assist device (LVAD) system as a bridge to transplant concept (Figure 2). Spontaneous left sided hemothorax due to thrombocytopenia and severe hemolysis under dual Impella therapy demanded a strategy change. Due to the unclear neurological status we were reluctant with a permanent LVAD support at this point. Hence, a temporary paracorporeal left ventricular assist in terms of a $36 \mathrm{~F}$ inflow cannula inserted via the left ventricular apex, a centrifugal pump (Rotaflow ${ }^{\circledR}$, Gettinge, Solna, Sweden) and an $19 \mathrm{~F}$ outflow cannula inserted into a $10 \mathrm{~mm}$ Dacron-Prothesis that was anastomosed to the ascending aorta was implanted. Simultaneously, the hemothorax was drained and the LV-Impella as well as the femoral VAECMO cannulas were explanted (Figure 3). 3 days later the RV-Impella was weaned and explanted. Under stable hemodynamic conditions the sedation was reduced, the patient remained adynamic but could move her extremities. Furthermore, a cerebral computer tomography (CT) showed no evidence of hypoxic sequelae or intracranial bleeding. Thus, a permanent LVAD (HeartMate $3^{\mathrm{TM}}$, Abbott, USA) was implanted on the $19^{\text {th }}$ day of her stay (Figure 4). 
Subsequently the patient gradually recovered, was responsive, opened her eyes and adequately moved her extremities. After extubation she suffered from epistaxis that needed nasal tamponade. On the $41^{\text {st }}$ day she was resuscitated for approximately 30 minutes following sudden hypotension, bradycardia and respiratory failure. Intubation and successful resuscitation bronchoscopy revealed severe lower airway obstruction by blood clots most likely due to epistaxis. Percutaneous tracheotomy was performed on the next day and cerebral CT scans showed no pathological findings. On the $59^{\text {th }}$ day the patient was successfully weaned and decannulated. Temporary right sided hemiparesis that had been observed following the resuscitation gradually resolved under intensive physiotherapy. Renal function also recovered so that renal replacement therapy was discontinued. On the $72^{\text {nd }}$ day an implantable cardioverter-defibrillator (Medtronic Evera MRI S VR SureScan) was implanted.

High-dose vasopressor therapy had led to pronounced acral necrosis of both feet. After initial conservative therapy with provision of temporary ortheses and frequent wound management, amputation of the I/IV digits of the left foot were performed in addition to an extensive debridement of necrotic tissue of both feet.

Frequent psychosomatic treatments focusing on strengthening resources, disease acceptance, disease insight, coping with acute somatic events and separation from the family were provided. She was transferred to a qualified rehabilitation facility with only minor neurological residues (limited mobility of the right leg) without any cognitive dysfunction after a total of 114 days after initial admission. She was discharged home on day 154.

\section{Discussion And Conclusions}

We report a case of a young woman with cardiogenic shock following severe coronary artery disease. Assumingly, the well-balanced combination of different options for ventricular support in early response to the individual course of the patient guided by an interdisciplinary team was the key to successful management of this extraordinary case (Table 1; Figure 1).

Mortality in cardiogenic shock is about 40\% [2]. In patients with acute heart failure and hemodynamic instability (INTERMACS level 1 und 2) short-term mechanical circulatory support (MCS) may be used to stabilize the patient, to gain time for further decisions on definitive therapy [1]. In a cohort of patients with cardiogenic shock in myocardial infarction Thiele identified a subgroup of $15-25 \%$ that will possibly survive by a MCS device, while 50-60 \% will survive without a MCS and 25-35\% will die with or without a MCS (e.g. brain death, sepsis) [11].

Different options for MCS are currently available. However, for a positive outcome the right choice of the device as well as proper timing for initiation as well as for escalation or de-escalation appear to be of paramount importance [5]. VA-ECMO provides complete cardiopulmonary support and is widely used in cardiothoracic surgery [5]. It is independent from the patient's rhythm and even in malignant arrhythmias will show no flow alterations [2]. However, VA-ECMO in it's peripheral (femoral) form may cause LV 
distension due to high afterload. LV distention in turn can exacerbate myocardial ischemia, trigger arrhythmias and may lead to pulmonary edema. Truby found $7 \%$ of patients to have a high and $22 \%$ to have considerable LV distension on VA-ECMO [12]. In the literature there are suggestions for LV unloading not only by inotropic support, but also by intra-aortic balloon pump (IABP), Impella and other surgical techniques [13].

A retrospective study by Pappalardo et al. showed a significantly lower in-hospital mortality (40\% vs. $74 \%)$ and a higher rate of a successful bridging to long-term strategies or recovery (62\% vs. $30 \%)$ in patients treated with concomitant VA-ECMO and Impella ${ }^{\circledR}$ as compared to treatment with VA-ECMO alone [6]. In the largest US-based retrospective study, VA-ECMO $(n=36)$ vs. ECPELLA $(n=30)$ in patients with refractory cardiogenic shock, the combined treatment with Impella ${ }^{\circledR}$ and VA-ECMO was associated with lower all-cause 30-day mortality, lower need for inotropic support and a comparable safety profile [7].

A metanalysis of 17 observational studies published in 2019 showed a lower mortality with different forms of LV unloading (54\% vs. $65 \%$ ), but in patients with additional Impella markedly more hemolysis was noted [14]. Therefore, the combination of VA-ECMO and Impella should be restricted to a limited period of time.

LV and RV Impella (BiPELLA) could be indicated in patients with a biventricular failure and contraindications to VA-ECMO. A simultaneous initiation of support with BiPELLA can be associated with improved survival outcomes [8]. A further research is required to provide evidence of the effectiveness of BiPELLA and an implantation of BiPELLA on top of a VA-ECMO.

The implantation of a temporary extracorporeal left ventricular assist device (e.g. Rotaflow ${ }^{\circledR}$ ) can bridge over a longer time period to a permanent device in unclear conditions. In the literature one case with a bridge to recovery is reported, in our case it was a bridge-to-bridge concept [15]. The most frequent complications are bleeding, thromboembolism and vascular complications, followed by neurological and respiratory problems [3].

A multidisciplinary heart-team (including cardiothoracic surgeons, cardiologists, anesthesiologists, intensive care specialists, and perfusionists) should join expertise to select and rapidly intervene on patients with severe cardiogenic shock $[9,10]$. Tehrani et al suggested the implementation of a multidisciplinary standardized team approach [10]. In their observational study a standardized team approach could significantly increase 30-day MCS survival from 47 to $77 \%$ [10]. We highly recommend the additional implementation of psychological and psychosocial support for the patient, the family and the team as well as access to ethical advice and palliative care as proposed in the European Guidelines and in the scientific statement of the American Heart Association [1].

In conclusion, the addition of LV Impella $\left(\mathrm{CP}^{\circledR}\right)$ implantation and RV support by Impella (RP $\left.{ }^{\circledR}\right)$ (BiPella) on top of VA-ECMO may support survival outcomes in refractory cardiogenic shock. In uncertain situations a paracorporal (short-term) LVAD (Rotaflow) can be used as a 'second bridge' to long-term mechanical support with LVAD. 


\section{Abbreviations}

AMI: acute myocardial infarction; BiPELLA: biventricular Impella; BTB: bridge-to-bridge; BTD: bridge to decision; BTR: bridge to recovery; BTT: bridge to transplantation; CRRT: continuous renal replacement therapy; CS: cardiogenic shock; CT: computer tomography; ECMO: extracorporeal membrane oxygenation; ECLS: Extracorporeal life support; 'ECPELLA' or 'ECMELLA': Impella ${ }^{\circledR}$ device to a veno-arterial extracorporeal membrane oxygenation; IABP: intra-aortal balloon pump; ICD: implantable cardioverterdefibrillator; LV: left ventricular; PROPELLA: prolonged Impella; Lp: lipoprotein; LVAD: left ventricular assist device; MCS: mechanical circulatory support; RV: right ventricular, VA-ECMO: veno-arterial extracorporeal membrane oxygenation;

\section{Declarations}

\section{Acknowledgements}

Not applicable.

\section{Author's contributions}

MS analyzed the patient data, drafted and edited this manuscript, provided the multidisciplinary ICU treatment as a psychosomatic medicine specialist. BG edited this manuscript, analyzed the patient data and provided the multidisciplinary ICU treatment as a cardiology specialist. AE-E edited this manuscript, was responsible for the multidisciplinary ICU treatment as a specialist for cardiothoracic surgery. L-OH, $\mathrm{OM}, \mathrm{KM}$ edited the manuscript and were responsible for the multidisciplinary ICU treatment as anesthesiology specialists. CJ edited the manuscript, was a cardiology specialist of the multidisciplinary ICU team and performed the impella implantation. HB edited the manuscript and performed the VA-ECMO and impella implantation, provided the multidisciplinary ICU treatment as a specialist for cardiothoracic surgery. IK performed the surgery and provided the multidisciplinary ICU treatment as a specialist for cardiothoracic surgery, edited this manuscript, and analyzed the patient data. The authors read and approved the final manuscript.

\section{Funding}

None.

\section{Availability of data and materials}

As a case report, all data generated or analyzed are included in this article. 


\section{Ethics approval and consent to participate}

The publication of this case report was allowed by the Ethics Committee of University of Göttingen Medical Center, Göttingen, Germany. Written informed consent was obtained from the patient reported in this study.

\section{Consent for publication}

The authors confirm that written consent for submission and publication of this case report including images and associated text has been obtained from the patient in line with COPE and CARE guidelines.

\section{Competing interests}

The authors have no conflicts of interest to declare in this work.

\section{References}

1. Ponikowski P, Voors AA, Anker SD, Bueno H, Cleland JGF, Coats AJS, et al. 2016 ESC Guidelines for the diagnosis and treatment of acute and chronic heart failure. Eur H J. 2016;37:2129-2200.

2. Mebazaa A, Combes A, van Diepen S, Hollinger A, Katz JN, Landoni G, et al. Management of cardiogenic shock complicating myocardial infarction. Intensive Care Med. 2018;44:760-73.

3. Thiele H, Jobs A, Ouweneel DM, Henriques JPS, Seyfarth M, Desch S, et al. Percutaneous short-term active mechanical support devices in cardiogenic shock: a systematic review and collaborative metaanalysis of randomized trials. Eur Heart J. 2017;38:3523-31.

4. Bellumkonda L, Gul B, Masri SC. Evolving concepts in diagnosis and management of cardiogenic shock. Am J Cardiol. 2018;122:1104-10.

5. Guglin A, Zucker MJ, Bazan VM, Bozkurt B, Banayosy AE, Estep JD, et al. Venoarterial ECMO for adults JACC scientific expert panel. JACC. 2019;73(6).

6. Pappalardo F, Schulte C, Pieri M, Schrage B, Contri R, Soeffker G, et al. Concomitant implantation of Impella ${ }^{\circledR}$ on top of veno-arterial extracorporeal membrane oxygenation may improve survival of patients with cardiogenic shock. Eur J Heart Fail. 2017;19:404-412.

7. Patel SM, Lipinski J, Al-Kindi SG, Patel T, Saric P, Li J, et al. Simultaneous venoarterial extracorporeal membrane oxygenation and percutaneous left ventricular decompression therapy with impella is associated with improved outcomes in refractory cardiogenic shock. ASAIO J. 2018;27:27.

8. Tschöpe C, Van Linthou S, Klein O, Mairinger T, Krackhardt F, Potapov EV, et al. Mechanical unloading by fulminant myocarditis: LV-IMPELLA, ECMELLA, BI-PELLA and PROPELLA concepts. $J$ of Cardiovasc Trans Res. 2019;12:116-123. 
9. Doll JA, Ohman EM, Patel MR, Milano CA, Rogers JG, Wohns DH, et al. A team-based approach to patients in cardiogenic shock. Catheter Cardiovasc Interv. 2016;88:424-433.

10. Tehrani BN, Truesdell AG, Sherwood MW, Shashank D, Tran HA, Epps KC, et al. Standardized teambased care for cardiogenic shock. J Am Coll Cardiol. 2019;73:1659-69.

11. Thiele H, Ohman EM, de Waha-Thiele S, Zeymer U, Desch S. Management of cardiogenic shock complicating myocardial infarction: an update 2019. Eur H J. 2019;40:2671-2683.

12. Truby LK, Takeda K, Mauro C, Yuzefpolskaya M, Garan AR, Kirtane AJ, et al. Incidence and implications of left ventricular distention during venoarterial extracorporal membrane oxygenation support. ASAIO J. 2017;63:257-265.

13. Meani P, Gelsomino S, Natour E, Johnson DM, Brunner La Rocca HP, Pappalardo F, et al. Modalities and effects of left ventricule unloading on extracorporal life support: a review of the current literature. Eur J Heart Fail. 2017;19:84-91.

14. Russo JJ, Aleksova N, Pitcher I, Couture E, Parlow S, Faraz M, et al. Left ventricular unloading during extracorporal membrane oxygenation in patients with cardiogenic shock. 2019;73:654-662.

15. Kashiwa K, Nishimura T, Saito A, Kubo H, Fukaya A, Tamai H, et al. Left heart bypass support with the Rotaflow Centrifugal Pump ${ }^{\circledR}$ as a bridge to decision and recovery in an adult. J Artif Organs. 2012;15:207-210.

\section{Figures}

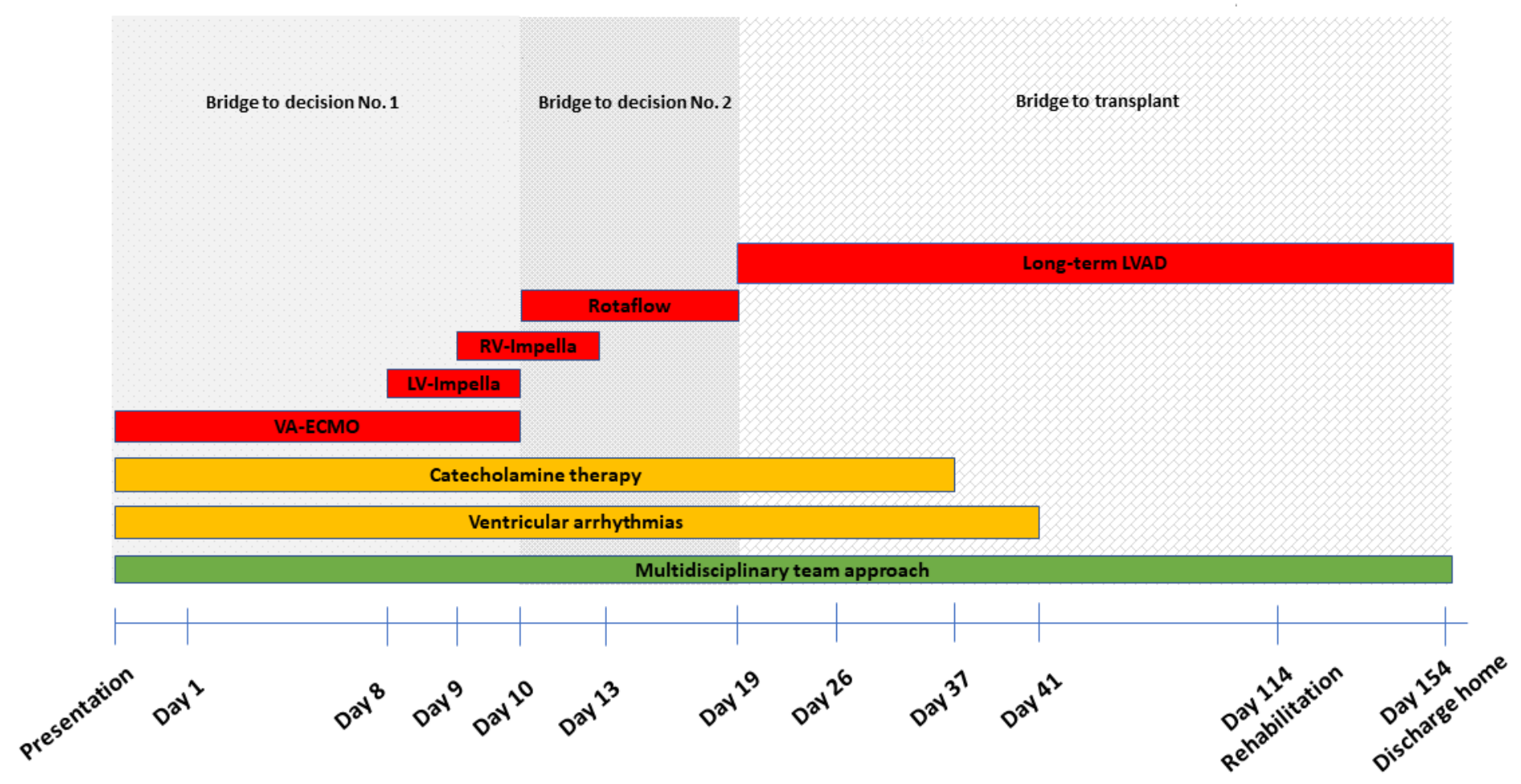


Timeline of devices, catecholamine therapy, ventricular arrhythmias, multidisciplinary team approach. VAECMO=veno-arterial extracorporeal membrane oxygenation, LV-Impella=left ventricular Impella, RVImpella=right ventricular Impella, LVAD=left ventricular assist device.

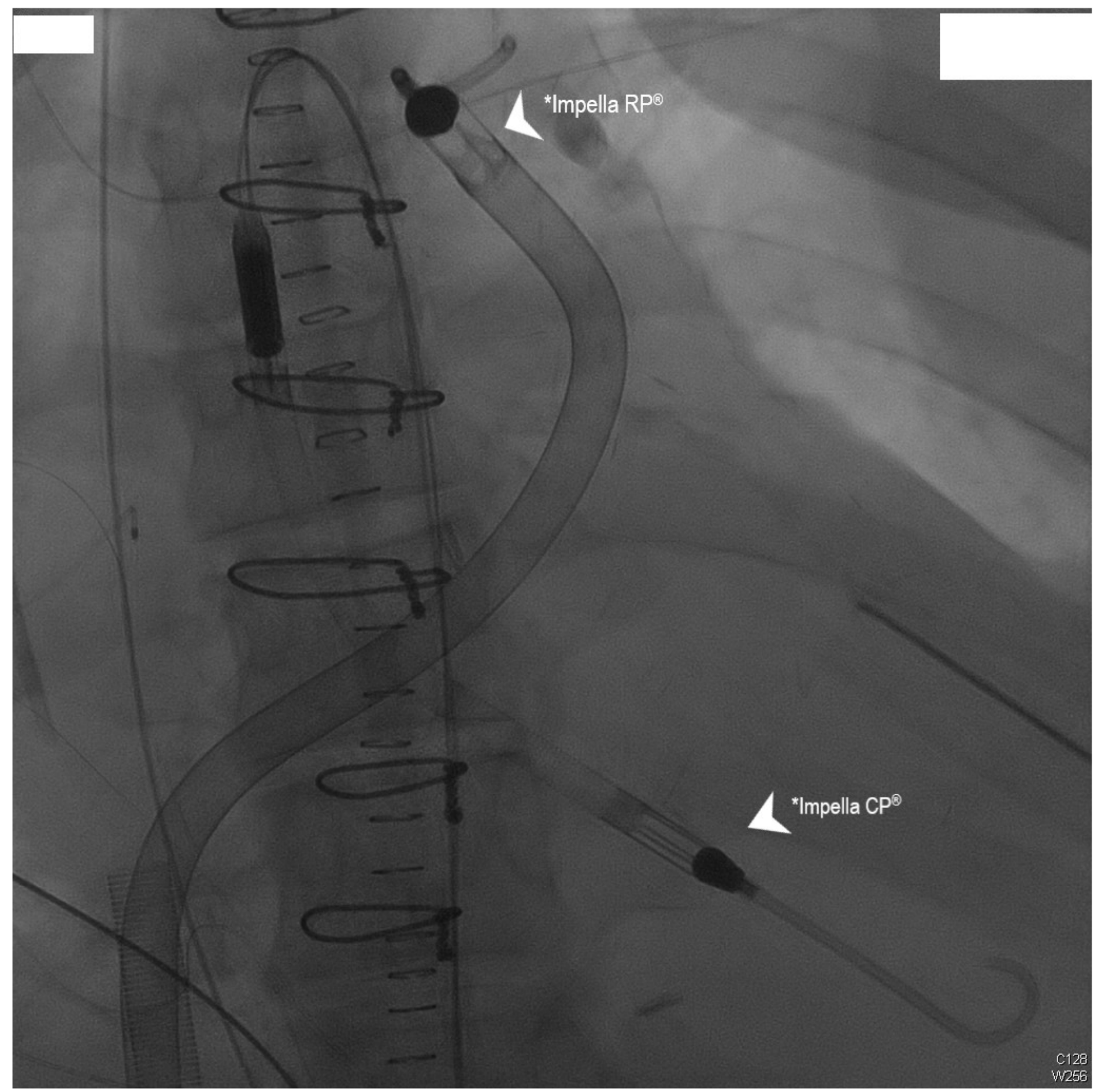

\section{Figure 2}

Impella $\mathrm{CP} \circledast$ and Impella $\mathrm{RP} \circledast$. *Impella $\mathrm{CP} \AA$ - Left ventricular Impella was inserted on the 8th day through a standard catheterization procedure through the femoral artery, into the ascending aorta, across the aortic valve into the left ventricle. *Impella RP® - Right ventricular Impella was inserted on the 9th day 
thought a standard catheterization procedure through the femoral vein and into the right atrium, across the tricuspid and pulmonic valves into the pulmonary artery.

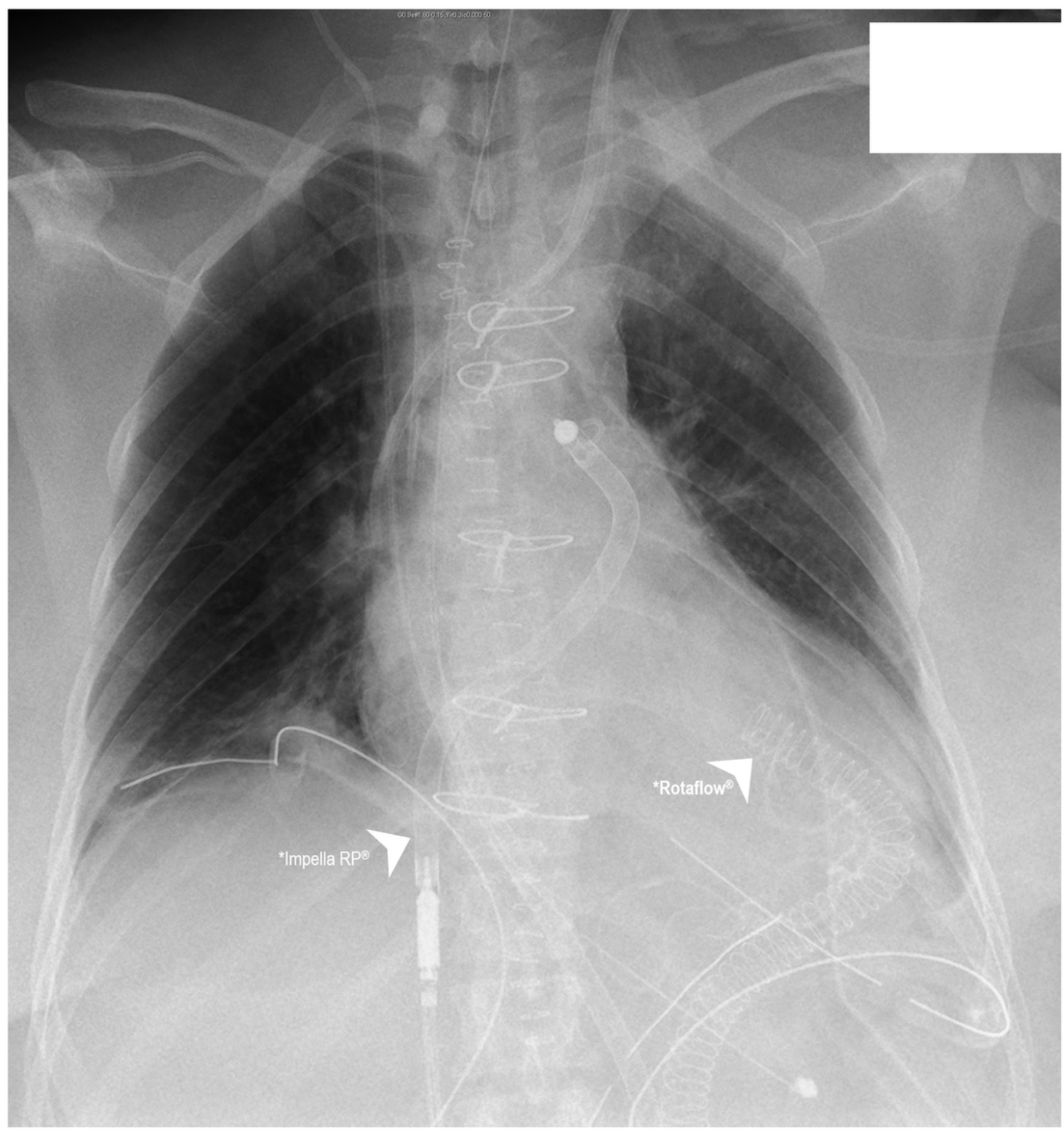

\section{Figure 3}

Impella RP® and Rotaflow. *Impella RP® - Right ventricular Impella. *Rotaflow ${ }^{\circledR}$ - Temporary paracorporeal left ventricular assist. A 36F inflow cannula was inserted on the 10th day via the left 
ventricular apex and a $19 \mathrm{~F}$ outflow cannula into a $10 \mathrm{~mm}$ Dacron-Prothesis that was anastomosed to the ascending aorta.

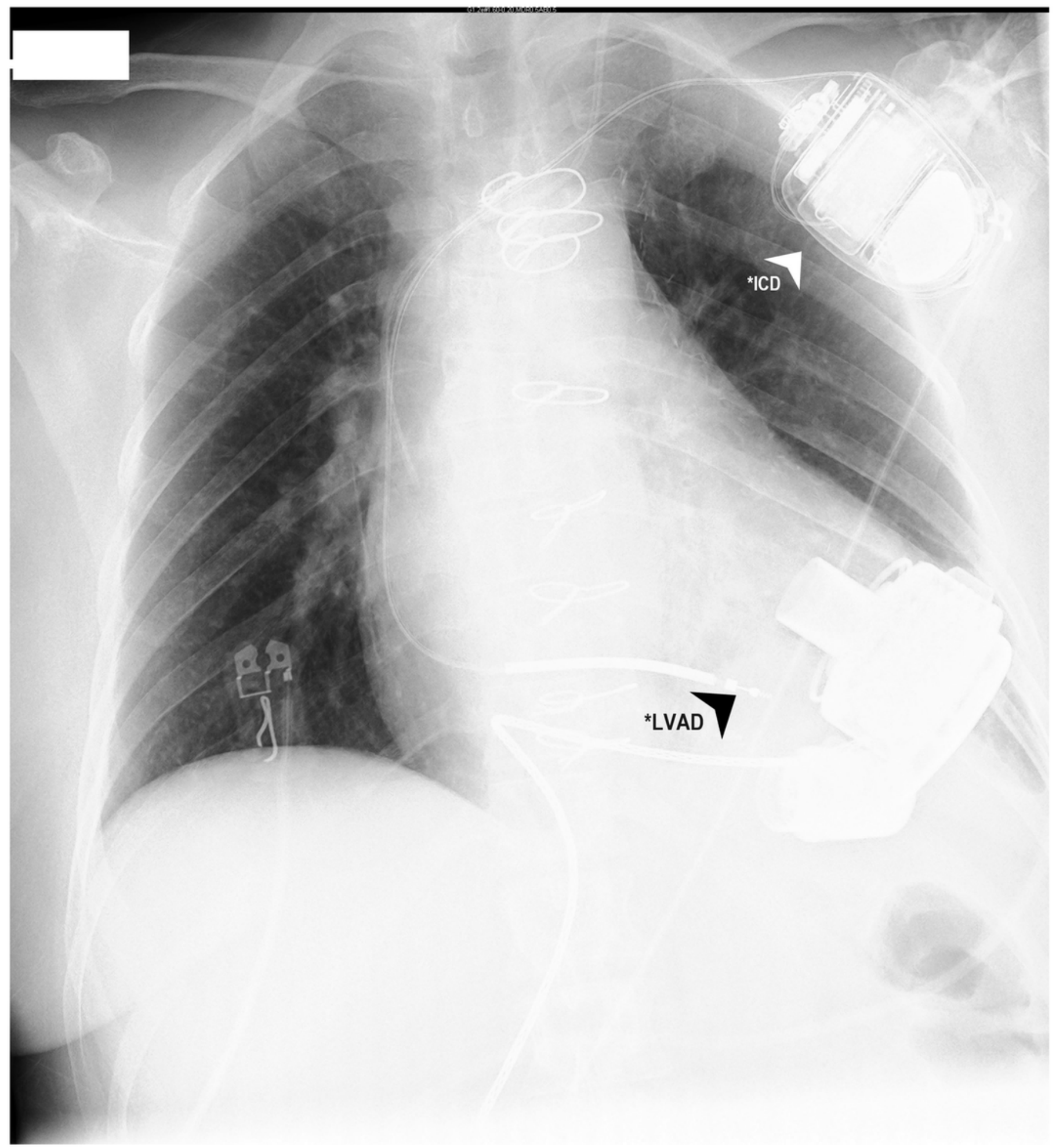

Figure 4

LVAD and ICD. *LVAD - Long-term left ventricular assist device (HeartMate 3TM) was implanted on the 19th day. ${ }^{*}$ ICD - implantable cardioverter-defibrillator (Medtronic Evera MRI S VR SureScan) was implanted pectoral left side on the day 72 . 


\section{Supplementary Files}

This is a list of supplementary files associated with this preprint. Click to download.

- CAREchecklistJournalofCardiothoracicSurgery.pdf 